\title{
Sinonasal outcome test-22 and peak nasal inspiratory flow - valuable tools in obstructive sleep apnoea*
}

\author{
Caroline Bengtsson', Lars Jonsson', Jenny Theorell-Haglöw², Mats \\ Holmström³, Christer Janson², Eva Lindberg² \\ ' Department of Surgical Sciences, Otorhinolaryngology, Head and Neck Surgery, Uppsala University, Uppsala, Sweden \\ ${ }^{2}$ Department of Medical Sciences, Respiratory, Allergy and Sleep Research, Uppsala University, Uppsala, Sweden \\ ${ }^{3}$ Division of Ear, Nose and Throat Diseases, Department of Clinical Sciences, Intervention and Technology, Karolinska University \\ Hospital, Huddinge, Sweden
}

Rhinology 58: 4, 341 - 348, 2020

https://doi.org/10.4193/Rhin19.189

*Received for publication:

May 8, 2019

Accepted: February 24, 2020

\begin{abstract}
Background: Sinonasal complaints contribute to low adherence to continuous positive airway pressure (CPAP) treatment. We aimed to investigate sinonasal health in obstructive sleep apnoea (OSA) patients, using the sinonasal outcome test-22 (SNOT-22), and to analyse whether SNOT-22 is affected by CPAP adherence. We also aimed to investigate whether peak nasal inspiratory flow (PNIF) was able to predict adherence to CPAP.
\end{abstract}

Methods: The study population comprised 197 OSA patients (60 females) initiating CPAP treatment. The SNOT-22, PNIF and the Epworth Sleepiness Scale were assessed at baseline and follow-up. One-night polygraphy, the Hospital Anxiety and Depression Scale, peak expiratory flow and health-related issues were assessed at baseline. At follow-up, the patients were categorised into adherent ( $>4$ hours/night) and non-adherent ( $<4$ hours/night) to CPAP treatment.

Results: The average time for following up CPAP treatment was (mean \pm SD) $24.0 \pm 23.9$ days and it did not differ significantly between the groups. The SNOT-22 score was elevated among all OSA patients, $36.1 \pm 19.4$. There was a larger improvement in the SNOT-22 score at follow-up among adherent CPAP users compared with non-adherent users (-10.4 \pm 13.9 vs. $-3.2 \pm 15.4)$. A PNIF value of $<100$ litres/min increased the risk of non-adherence to CPAP with an adjusted odds ratio (OR) of $2.40((95 \% \mathrm{Cl} 1.16-5.00))$.

Conclusions: The SNOT-22 was elevated in patients with OSA, indicating a considerable sinonasal disease burden. The SNOT-22 improved with good CPAP adherence. A low PNIF value was able to predict poor CPAP adherence. Both the SNOT-22 and PNIF can be valuable tools in the evaluation of OSA patients and in the management of CPAP treatment.

Key words: CPAP, nasal obstruction, OSA, PNIF, SNOT-22

\section{Introduction}

Sinonasal complaints are prevalent among patients with obstructive sleep apnoea (OSA) ${ }^{(1-3)}$ and subjective nasal obstruction was reported among 35\% of OSA patients in a recent study ${ }^{(4)}$. In addition, reduced objective nasal measurements and lower nasal patency among OSA patients compared with controls have been reported ${ }^{(5)}$.

Although prevalent, sinonasal complaints among OSA patients have been sparsely investigated using sinonasal health-related quality of life instruments. The most suitable, validated, healthrelated quality of life instrument to assess sinonasal disease is the sinonasal outcome test-22 (SNOT-22) ${ }^{(6)}$.
The versatility of the SNOT-22 makes it suitable for the evaluation of sinonasal issues, as well as a wide range of other healthrelated issues. To our knowledge, there is only one OSA study, which included 30 patients, in which the SNOT-22 has been used ${ }^{(7)}$. The total SNOT-22 score was elevated compared with controls and several sinonasal complaints were correlated with OSA severity.

Continuous positive airway pressure (CPAP) is the standard treatment worldwide for moderate to severe OSA. Adherence rates are poor, however, and nasal obstruction is a contributory factor (8). Consequently, a substantial percentage of OSA patients do not use their CPAP and therefore run an increased risk of long- 
term health consequences ${ }^{(9)}$.

To improve CPAP adherence rates, it would be desirable to have a tool which was able to identify patients with nasal obstruction who may be at risk of low adherence. Initial CPAP treatment could then be personalised and optimised, with improved adherence as a result.

Peak nasal inspiratory flow (PNIF) is a well-recognised, inexpensive, portable, quick and easy-to-use tool for evaluating nasal patency ${ }^{(10)}$. It has been used in a wide range of studies and has proven to be reliable, with high reproducibility ${ }^{(10-13)}$.

The aim of the present study was to investigate sinonasal health in 200 consecutive CPAP-naïve OSA patients using the SNOT-22 and to analyse whether the SNOT-22 is affected by CPAP adherence. We also aimed to investigate whether PNIF was able to predict adherence to CPAP.

\section{Methods}

The study was conducted at the Centre of Sleep and Breathing at Uppsala University Hospital. Patients aged 18 to 80 years, diagnosed with OSA and prescribed CPAP treatment, were consecutively recruited to the study in connection with the initiation of CPAP treatment. The baseline visit was then followed by a second visit, according to the standard clinical protocol, approximately 3-4 weeks later (number of days, mean 24.0 \pm 23.9 SD) (Table 1). The cohort was subsequently divided into two groups, based on adherence to CPAP. An average use of CPAP treatment of $>4$ hours/night was regarded as adherent, while $<4$ hours/ night was non-adherent ${ }^{(14)}$. See Figure 1 for a study flow chart. At baseline and at follow-up, PNIF was measured with a portable Youlten Peak Nasal Inspiratory Flow Meter ${ }^{\circledast}$ (Clement Clarke International Ltd, Harlow, UK), range 0-370 litres/minute. After having become acclimatised to the indoor room temperature for $\geq 30$ minutes, all the patients were tested in a sitting position $(15,16)$. At the end of a full expiration, the patients were instructed to inhale as quickly and forcefully as they could through the nose, with the mouth closed and with the mask firmly over the face. Three satisfactory inspirations were obtained and the highest PNIF value was used in the statistical analyses. All the assessments were supervised by trained nurses.

As part of the clinical sleep apnoea investigation, patients also performed peak expiratory flow (PEF), to measure lower respiratory tract function. This was performed in a sitting or standing position two or three times with a Mini-Wright ${ }^{\mathrm{TM}}$ (Clement Clarke International Ltd, Harlow, UK) standard range peak flow meter.

Sinonasal problems and related symptoms were documented at baseline and follow-up using the disease-specific, healthrelated, quality of life instrument, the SNOT-22 ${ }^{(17)}$. This instrument was developed for use in chronic rhinosinusitis (CRS) with or without nasal polyposis. It encompasses 22 questions, on not

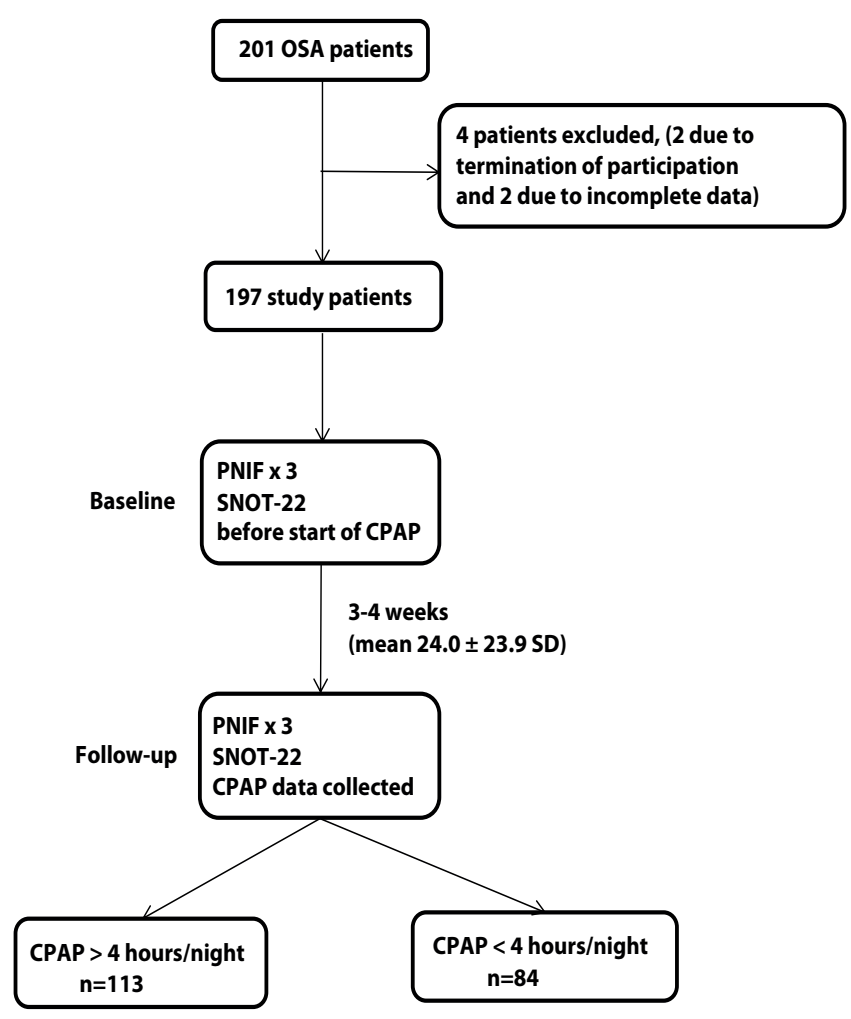

Figure 1. Flow chart of the study. CPAP - continuous positive airway pressure, OSA - obstructive sleep apnoea, PNIF - peak nasal inspiratory flow, SNOT-22 - sinonasal outcome test 22.

only patient-reported sinonasal complaints but also other complaints, including physical problems, functional limitations and emotional consequences ${ }^{(18)}$. It is subcategorised into the four domains of 'rhinologic' (seven questions), 'ear/facial' (five questions), 'sleep' (four questions) and 'psychologic' (six questions), which improves the precision when interpreting the data ${ }^{(19)}$. The question "How bothersome has each of the following problems from the nose/sinuses been to you during the past two weeks?" was answered by the response options $0=$ no problem, $1=$ very mild problem, $2=$ mild or slight problem, $3=$ moderate problem, $4=$ severe problem, $5=$ problem as bad as it can be. The seven problems of the rhinologic domain were'need to blow nose', 'sneezing,' 'runny nose,' 'blockage/congestion of nose', 'loss of sense of taste/smell', 'post-nasal discharge (dripping at the back of your nose)' and 'thick nasal discharge'. The five problems of the ear/facial domain included 'cough', 'ear fullness', 'dizziness,',ear pain' and 'facial pain/pressure'. The four problems of the sleep domain were 'difficulty falling asleep', 'waking up at night', 'lack of a good night's sleep' and 'waking up tired'. The six problems of the psychologic domain were 'fatigue', 'reduced productivity,',reduced concentration', 'frustrated/restless/ irritable', 'sad' and 'embarrassed'. The total score ranges from 0-110 points, with a low score indicating better quality of life. The minimal clinically important difference is 8.9 points ${ }^{(17)}$. The 
Table 1. Characteristics of the population. Data are presented as $n(\%)$ and the mean \pm SD. Percentages relate to the number of patients who answered the question positively.

\begin{tabular}{|c|c|c|c|c|}
\hline & $\begin{array}{c}\text { All } \\
(n=197)\end{array}$ & $\begin{array}{c}\text { Adherent } \\
>4 \text { hours/night } \\
\text { ( }=113)\end{array}$ & $\begin{array}{c}\text { Non-adherent } \\
<4 \text { hours/night } \\
(n=84)\end{array}$ & $\mathbf{p}$ \\
\hline Age (years) & $57.7 \pm 11.3$ & $57.2 \pm 11.1$ & $58.5 \pm 11.7$ & 0.42 \\
\hline Gender (male) & $137(69.5)$ & $77(68.1)$ & $60(71.4)$ & 0.62 \\
\hline BMI $\left(\mathrm{kg} / \mathrm{m}^{2}\right)$ & $31.8 \pm 5.7$ & $31.3 \pm 5.0$ & $32.4 \pm 6.5$ & 0.18 \\
\hline Smoking & $18(9.7)$ & $10(9.3)$ & $8(10.3)$ & 0.82 \\
\hline CRS symptoms & $46(23.4)$ & $21(18.6)$ & $25(29.8)$ & 0.07 \\
\hline Allergic rhinitis & $31(16.4)$ & $19(17.6)$ & $12(14.8)$ & 0.61 \\
\hline HAD anxiety & $5.4 \pm 3.6$ & $5.2 \pm 3.7$ & $5.7 \pm 3.6$ & 0.37 \\
\hline HAD depression & $4.9 \pm 3.3$ & $4.7 \pm 3.2$ & $5.3 \pm 3.3$ & 0.19 \\
\hline PEF (I/min) & $450.9 \pm 118.0$ & $465.1 \pm 129.2$ & $431.5 \pm 98.8$ & 0.14 \\
\hline Asthma & $22(13.3)$ & $16(16.5)$ & $6(8.7)$ & 0.14 \\
\hline Hypertonia & $98(55.4)$ & $52(52.0)$ & $46(59.7)$ & 0.30 \\
\hline Diabetes & $34(20.0)$ & $13(13.5)$ & $21(28.4)$ & 0.02 \\
\hline Heart disease & $38(23.2)$ & $19(20.0)$ & $19(27.5)$ & 0.26 \\
\hline Gastroesophageal reflux & $60(36.4)$ & $35(36.8)$ & $25(35.7)$ & 0.88 \\
\hline Headache & $62(39.7)$ & $37(40.7)$ & $25(38.5)$ & 0.78 \\
\hline Joint pain & $63(36.6)$ & $32(32.3)$ & $31(42.5)$ & 0.17 \\
\hline Septal surgery & $16(8.2)$ & $7(6.25)$ & $9(10.7)$ & 0.26 \\
\hline Nasal polyp surgery & $8(4.1)$ & $4(3.6)$ & $4(4.8)$ & 0.68 \\
\hline Nasal fracture surgery & $6(3.1)$ & $4(3.6)$ & $2(2.4)$ & 0.63 \\
\hline RF conchotomy & $2(1.0)$ & $1(0.9)$ & $1(1.2)$ & 0.84 \\
\hline Other nasal surgery (plastic or tumour surgery) & $5(2.6)$ & $3(2.7)$ & $2(2.4)$ & 0.90 \\
\hline Nasal medication PNIF 1 & $31(16.0)$ & $20(18.2)$ & $11(13.1)$ & 0.34 \\
\hline Nasal medication PNIF 2 & $37(20.3)$ & $27(25.0)$ & $10(13.5)$ & 0.06 \\
\hline CPAP use, hours/night & $4.65 \pm 2.47$ & $6.37 \pm 1.26$ & $1.96 \pm 1.11$ & $<0.001$ \\
\hline Number of days between baseline and follow-up & $24.0 \pm 23.9$ & $22.0 \pm 20.4$ & $26.9 \pm 28.0$ & 0.17 \\
\hline
\end{tabular}

BMI - Body Mass Index, CPAP - Continuous Positive Airway Pressure, CRS - Chronic rhinosinusitis, HAD - Hospital Anxiety and Depression Scale, PEF - Peak Expiratory Flow, I/min - litres/minute, RF -radiofrequency.

mean total SNOT-22 score and the mean score for each domain were calculated at baseline and at follow-up for those who had complete data on both occasions. The total SNOT-22 delta score was also calculated.

The Hospital and Anxiety Depression (HAD) scale was used to evaluate anxiety and depression at baseline ${ }^{(20)}$. This validated questionnaire consists of 14 questions, seven on anxiety and seven on depression. The highest score is 42 points. A score of $\geq$ 8 in either category is considered to indicate possible disease. The Epworth Sleepiness Scale (ESS) ${ }^{(21)}$ was used to evaluate excessive daytime sleepiness at baseline and at follow-up. This is a validated questionnaire evaluating the risk of dozing off in eight different passive, daytime situations. The total score ranges from 0-24 and a score of $>10$ is considered to indicate excessive daytime sleepiness. Delta-ESS was calculated as ESS at baseline subtracted from ESS at follow-up.
Baseline information on other diseases and objective sleep variables was collected from medical records, as well as the ambulatory polygraphy (Nox T3, Nox Medical, Reykjavik, Iceland) results of the standardised obstructive sleep apnoea investigation of each patient. The polygraphy recording included measurements of saturation by pulse oximetry, respiratory movements by thoracic and abdominal belts and nasal airflow by a nasal pressure sensor and snoring by a microphone. The polygraphy report (Noxturnal, Nox Medical, Reykjavik, Iceland) was scored by nurses specialised in sleep apnoea scoring. A questionnaire on symptoms related to CPAP treatment was also used at follow-up. Adherence data were downloaded from the CPAP memory card. The study was approved by the regional ethical review board in Uppsala, Dnr 2014/189. Written consent was obtained from all the patients in the study. 
Table 2. Polygraphy results and CPAP data for obstructive sleep apnoea (OSA) and results of the Epworth Sleepiness Scale (ESS). Data are presented as $\mathrm{n}(\%)$ and the mean $\pm \mathrm{SD}$.

\begin{tabular}{|c|c|c|c|c|}
\hline & $\begin{array}{c}\text { All } \\
(n=197)\end{array}$ & $\begin{array}{c}\text { Adherent } \\
>4 \text { hours/night } \\
(n=113)\end{array}$ & $\begin{array}{c}\text { Non-adherent } \\
<4 \text { hours/night } \\
(n=84)\end{array}$ & $\mathbf{p}$ \\
\hline \multicolumn{5}{|l|}{ OSA severity } \\
\hline $\mathrm{AHI}<5$ & $1(0.5)$ & $1(0.9)$ & $0(0.0)$ & 0.59 \\
\hline AHI 5-14.9 & $35(17.8)$ & $18(15.9)$ & $17(20.2)$ & \\
\hline AHI 15-29.9 & $71(36.0)$ & $39(34.5)$ & $32(38.1)$ & \\
\hline $\mathrm{AHI} \geq 30$ & $90(45.7)$ & $55(48.7)$ & $35(41.7)$ & \\
\hline AHI mean & $31.8 \pm 19.1$ & $33.4 \pm 20.4$ & $29.8 \pm 17.2$ & 0.19 \\
\hline ODI mean & $29.3 \pm 18.8$ & $30.3 \pm 20.0$ & $27.9 \pm 17.0$ & 0.39 \\
\hline CPAP pressure, $\mathrm{cm} \mathrm{H}_{2} \mathrm{O}$ & $11.6 \pm 1.5$ & $11.6 \pm 1.4$ & $11.6 \pm 1.6$ & 0.98 \\
\hline $\mathrm{AHI}<5$, at follow-up & $154(78.2)$ & $100(88.5)$ & $54(64.3)$ & $<0.001$ \\
\hline $\mathrm{AHI}$ at follow-up & $2.94 \pm 4.04$ & $2.65 \pm 2.95$ & $3.43 \pm 5.40$ & 0.21 \\
\hline Minimum saturation (\%) & $77.2 \pm 8.0$ & $76.5 \pm 8.5$ & $78.2 \pm 7.2$ & 0.13 \\
\hline Mean saturation (\%) & $92.1 \pm 2.1$ & $92.0 \pm 2.1$ & $92.3 \pm 2.0$ & 0.25 \\
\hline ESS at baseline & $10.5 \pm 4.7$ & $10.7 \pm 4.8$ & $10.2 \pm 4.6$ & 0.44 \\
\hline Delta ESS & $-2.3 \pm 5.0$ & $-3.7 \pm 5.3$ & $-0.4 \pm 3.7$ & $<0.001$ \\
\hline
\end{tabular}

AHI - Apnoea-Hypopnoea Index per hour, CPAP - continuous positive airway pressure, ODI - Oxygen Desaturation Index.

\section{Statistical analyses}

Stata 12.1 (Stata Corp, TX, USA) was used in all the statistical analyses. The unpaired t-test and $\chi^{2}$ test were used to perform univariate analyses. The results were presented as $\mathrm{n}(\%)$ and the mean $\pm(S D)$. The null hypothesis was rejected at a level of $p<0.05$. In line with previous publications on the SNOT-22, simple mean imputation was used for missing data ${ }^{(17,22)}$. This was applied when at least $50 \%$ of the items were completed and it means that the value of the missing data was replaced by the mean of the values of the completed item. The PNIF values were divided into quartiles. Multiple regression analysis was performed to analyse the association between the lowest PNIF quartile and non-adherence.

\section{Definitions}

Body mass index (BMI) was calculated as measured body weight ( $\mathrm{kg}$ ) divided by height $(\mathrm{m})$ squared $\left(\mathrm{kg} / \mathrm{m}^{2}\right)$.

Smoking was defined as a positive answer to the question "Do you smoke?".

Chronic rhinosinusitis (CRS) was defined according to the epidemiological symptom criteria in the European Position Paper on Rhinosinusitis and Nasal Polyps (EPOS) ${ }^{(23)}$. This definition is based only on symptoms and does not include an objective assessment of the nose or sinuses. The patients had to confirm the presence of two or more of the following symptoms during the last 12 months: a) nasal blockage/obstruction/congestion, b) nasal discharge (anterior/posterior nasal drip), c) facial pain or pressure and d) reduction in or loss of smell. At least one symptom had to be nasal blockage or nasal discharge and all the symptoms needed to have a minimum symptom duration of $\geq 12$ weeks.

Allergic rhinitis was defined as a positive answer to both of the questions "Do you have allergic rhinitis or any other nasal allergy?" and "Have you had problems with allergic rhinitis during the past 12 months?".

Previous and/or present asthma, hypertonia, diabetes, heart disease, gastroesophageal reflux, headache or joint pain was reported in a clinical questionnaire (Yes/No).

Nasal medication was defined by a positive answer to the question, which was asked in connection with the PNIF measurements, "Have you used any nasal medication during the past six to eight hours?" and the confirmation of any or all of the following medical options: nasal decongestant spray, nasal steroid spray and antihistamine tablet.

The apnoea-hypopnoea index (AHI) was divided into four categories based on generally applied clinical thresholds: 0 to $<5$, 5-14.9, 15-29.9 and $\geq 30$ events/hr. An apnoea was defined as a $>90 \%$ reduction in nasal airflow lasting more than $\geq 10$ seconds. A hypopnoea was defined as a $30-90 \%$ reduction in nasal airflow over $\geq 10$ seconds with a desaturation of $\geq 4 \%$ measured by pulse oximetry.

The oxygen desaturation index (ODI) was defined as the number of desaturations per hour of $\geq 4 \%$, as measured by pulse oximetry. 


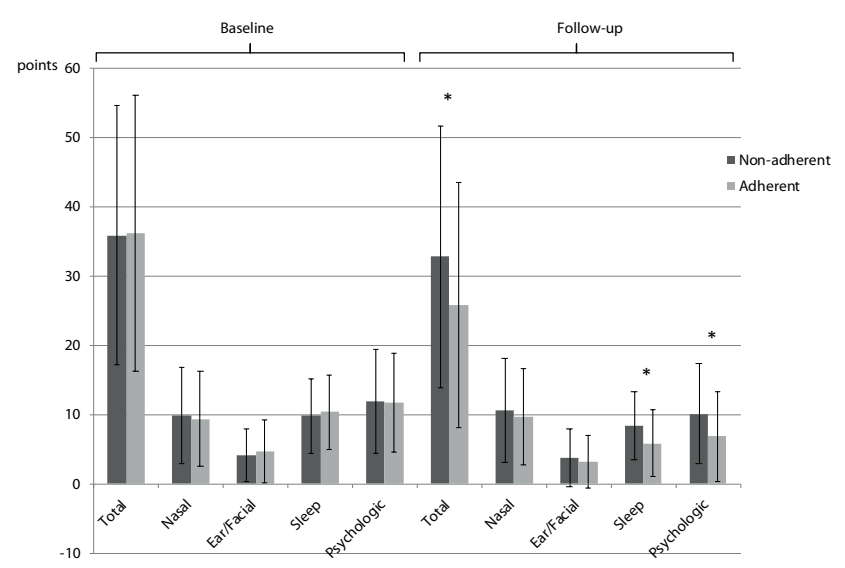

Figure 2. Sinonsasal outcome test-22 (SNOT-22) results at baseline and follow-up for the adherent and the non-adherent group. ${ }^{*} \mathrm{p}<0.05$.

\section{Results}

Study population

A total of 201 patients were recruited to the study. Two patients terminated their participation before follow-up. Data were missing for another two patients, rendering a total study population of 197 patients, 60 females (30.5\%) and 137 (69.5\%) males with a mean age of 57.7 years. Of the 197 patients, 113 were adherent CPAP users, while 84 were non-adherent users. Population characteristics are presented in Table 1.

For the whole population the mean BMI was $31.8 \mathrm{~kg} / \mathrm{m}^{2}$. Smoking had a prevalence of $9.7 \%$. The non-adherent and adherent groups were similar in terms of these variables and the average number of days between the baseline and follow-up visits. Both groups had a prevalence of allergic rhinitis similar to that of the total population (Table 1).

Diabetes was more common among non-adherent CPAP users. The other analysed diseases did not differ between the two groups, nor did the prevalence of previous nasal surgery or use of nasal medication at baseline and at follow-up (Table 1).

\section{Obstructive sleep apnoea}

The adherent and non-adherent CPAP users did not differ significantly in terms of OSA severity (Table 2). Severe OSA with an $\mathrm{AHI}$ of $\geq 30$ had a prevalence of $48.7 \%$ in the adherent group and $41.7 \%$ in the non-adherent group. A total of $81.7 \%$ of the patients had moderate or severe OSA, equivalent to at least an $\mathrm{AHI}$ of $>15$.

Approximately $74 \%$ of patients used a nasal mask from the start of treatment (no difference between the adherent and nonadherent groups) and the remainder of the study groups was equipped with a full face mask or, in a few cases, both. In the non-adherent group, 39.5\% experienced mask discomfort at follow-up compared with $6.5 \%$ in the adherent group. At followup, $44.0 \%$ of the non-adherent users switched to a different mask model compared with $27.4 \%$ in the adherent group. Mask
Table 3. Risk factors for low CPAP adherence in the total population.

\begin{tabular}{|c|c|c|c|}
\hline & $\begin{array}{c}\text { CPAP adherence } \\
<4 \text { hours/night } \\
\text { (n=178) }\end{array}$ & & \\
\hline & OR & $95 \% \mathrm{Cl}$ & $p$ \\
\hline $\begin{array}{l}\mathrm{PNIF}<100 \mathrm{I} / \mathrm{min} \text {, } \\
\text { at baseline }\end{array}$ & 2.40 & $1.16-5.00$ & 0.02 \\
\hline Age (years) & 1.02 & $0.99-1.05$ & 0.28 \\
\hline Gender (male) & 0.72 & $0.36-1.44$ & 0.36 \\
\hline BMI $\left(\mathrm{kg} / \mathrm{m}^{2}\right)$ & 1.04 & $0.99-1.11$ & 0.14 \\
\hline Smoking & 0.60 & $0.21-1.71$ & 0.34 \\
\hline
\end{tabular}

The results are presented as the OR $(95 \% \mathrm{Cl})$ after adjusting for all the variables in the table. Calculations were made for all patients with complete data on the variables in the table. BMI - Body Mass Index, CPAP - Continuous Positive Airway Pressure, I/min - litres/minute, PNIF - Peak Nasal Inspiratory Flow.

leakage was experienced by both groups, but the adherent group had a higher percentage of low mask leakage ( $<5$ litres/ minute) than the non-adherent group, $63.7 \%$ vs $36.9 \%$. High mask leakage ( $>24$ litres/minute) was, however, more common in the adherent group compared with the non-adherent group, $21.5 \%$ vs $10.6 \%$. A humidifier was added to the CPAP treatment at follow-up in $46 \%$ of the patients in both groups (data not shown).

\section{Epworth Sleepiness Scale (ESS)}

The mean ESS score in the total population at baseline was 10.5 $\pm 4.7 \mathrm{SD}$ (Table 2). The score was similar in the two groups. At follow-up, there was an improvement in the mean ESS score in both groups, which differed significantly from the baseline score. The largest improvement was in the adherent group, with an average reduction of 3.7 points.

\section{SNOT-22}

At baseline, 15 patients had fewer than $50 \%$ of completed items on the SNOT-22 and were therefore excluded from the calculations. At follow-up, 21 patients were excluded due to incomplete data, leaving 168 patients with complete SNOT-22 data at both baseline and follow-up. The total mean SNOT-22 score at baseline in the total population was $36.1 \pm 19.4$ SD and the scores were on the same level for the adherent and non-adherent groups. In addition, the subdomain scores did not differ between the two adherence groups at baseline (Figure 2). The adherent group had an improved and significantly lower total SNOT-22 score compared with the non-adherent group at follow-up. The sleep and psychologic domain scores improved the most for both groups, but the improvement was larger in the adherent group (Figure 2). Consequently, the delta SNOT-22 
score was reduced to a greater extent for the adherent CPAP users, compared with the non-adherent users, (mean $=-10.4 \pm$ 13.9 SD versus $-3.2 \pm 15.4 S D ; p=0.002$ ).

\section{Peak nasal inspiratory flow}

A PNIF value in the lowest quartile $(<100 \mathrm{l} / \mathrm{min})$ at baseline was associated with a 2.24 risk of non-adherence. After adjusting for age, BMI, gender and smoking, the adjusted odds ratio was 2.40 for non-adherence. Age, BMl, gender and smoking did not appear to predict non-adherence (Table 3).

The mean \pm SD PNIF values at baseline for females and males in the adherent group were $130.0 \pm 49.6$ and $175.5 \pm 68.3$ litres/ min respectively. In the non-adherent group, the corresponding values were $134.4 \pm 65.0$ for females and $157.6 \pm 77.2$ for males. The mean \pm SD PNIF for the adherent group as a whole was $161.0 \pm 66.3$ litres/min, and for the non-adherent group $151.0 \pm$ 74.3 litres/min, $(p=0.32)$. After having excluded all patients who had used nasal medication (nasal decongestant spray, nasal steroid spray or antihistamine tablet) the corresponding results for the adherent group $(n=90)$ were 151.2 \pm 77.4 , and the nonadherent group $(n=73) 166.4 \pm 67.0,(p=0.18)$. The delta PNIF did not differ significantly between the two groups and nor did PNIF at baseline when comparing smokers with non-smokers (data not shown).

\section{Discussion}

The main finding in the present study was that OSA patients had a high average SNOT-22 score and the SNOT-22 score improved with good CPAP adherence. We also found that a low PNIF value ( $<100 \mathrm{l} / \mathrm{min}$ ), a sign of nasal obstruction, was able to predict non-adherence to initial CPAP.

The total mean SNOT-22 score at baseline among all OSA patients was $36.1 \pm 19.4$ SD, with similar score levels in both the adherent and the non-adherent group. The scores were generally higher compared with the results of a recent original and review study by Farhood et al. ${ }^{(24)}$. They reported a SNOT-22 score of $11 \pm 9.4$ among healthy individuals without sinonasal disease. They also found that asthma and depression are associated with a higher total SNOT-22 score. As our patients had normal HAD scores and an asthma prevalence of $13 \%$, close to the Swedish national level of $10 \%{ }^{(25)}$, the impact of these diseases on the SNOT-22 score appears to be limited.

Sinonasal symptoms may partly explain the high SNOT-22 scores in our study, since nasal obstruction affects approximately $35 \%$ of OSA patients ${ }^{(4)}$. The cardinal symptoms of CRS include nasal obstruction, nasal secretion, facial pressure and lack of smell. Furthermore, CRS symptoms affects approximately $8.4 \%$ of the adult Swedish population ${ }^{(26)}$. In the present study, $23.4 \%$ of the total study population had symptoms of CRS and the total SNOT-22 scores were equal to those of patients with CRS, with or without nasal polyposis, six and 12 months after surgery ${ }^{(27)}$.
The role of sinonasal symptoms in the high total SNOT-22 score is further emphasised when comparing our results with those of Kuan et al. ${ }^{(7)}$. In their study of 30 OSA patients, diagnosed with polysomnography and without sinonasal disease, the average SNOT-22 score was $29.1 \pm 3.3$. Our average SNOT-22 score was $36.1 \pm 19.4$, which is partly explained by a higher rhino domain score in the present study $(9.4 \pm 6.8$ vs $4.9 \pm 1.0)$. Furthermore, our patients had more severe OSA disease, with a mean AHI of 32 /hour compared with 25 /hour in the study by Kuan et al. This most probably generated higher average scores in the other questionnaire domains. The difference in sample size between the two studies may also play a role in disparities in results. Our findings emphasise the importance of evaluating and treating sinonasal disease among OSA patients. The results also acknowledge sleep quality and psychological issues as important variables in treating OSA patients. Indeed, in line with our findings, daytime sleepiness in patients investigated for sleepdisordered breathing has been associated with several patientreported complaints, but not with PNIF and self-reported nasal obstruction ${ }^{(28)}$. The improved scores in the sleep and psychological domains of the SNOT-22 and ESS score at follow-up among adherent CPAP users clearly underline this notion. It also suggests that the SNOT-22 could be used as a tool in the follow-up of CPAP treatment. This, however, requires further investigation. There was a 2.40 increase in the risk of non-adherence to CPAP among patients with a PNIF value of $<100 \mathrm{l} / \mathrm{min}$. The association between a low PNIF value, indicating impaired nasal breathing, and low CPAP adherence is consistent with previous findings ${ }^{(29-31)}$. Various factors may influence the PNIF value. It decreases with age and is lower in females ${ }^{(16)}$. In the present study, the adherent and non-adherent groups did not differ in terms of age and gender. PNIF improves with practice, which is why the best of three PNIF values was used in the present study, but it does not differ significantly in the upright or sitting position ${ }^{(15,16)}$. The mean PNIF values for females and males in the adherent and non-adherent groups were in the upper normal ranges compared with the results in healthy subjects without nasal problems and OSA ${ }^{(16,32-34)}$. Differences in the number of patients, age span and ethnicity may affect the PNIF values and thereby explain the wide normal intervals.

We found a higher prevalence of diabetics among those non-adherent to CPAP. In our opinion there may be a tendency among physicians that diagnose OSA to prescribe CPAP treatment somewhat more generously to patients with diabetes, since this is an associated disease with OSA, and prevention of comorbid conditions is essential. This may have contributed to an over representation of diabetics with milder OSA in the present study. Also, diabetics may have a treatment that involves practical challenges with daily measurements of blood glucose, injections of insulin, tablet intake, food restrictions etc. The addition of CPAP treatment to other treatments may be experienced as 
an additional burden, making diabetics more likely to become non-adherent to CPAP ${ }^{(35)}$.

One advantage with the present study was the inclusion of 201 consecutive OSA patients, who were examined in a regular clinical setting. In addition, OSA was diagnosed with one-night ambulatory polygraphy according to national guidelines. Validated questionnaires such as the SNOT-22 and ESS were used and CRS was defined according to the EPOS epidemiological symptom criteria, making the results comparable with those in other studies. Furthermore, according to the Swedish sleep apnoea registry, Sesar ${ }^{(36)}$, and the Swedish national registry for CPAP treatment, Swedevox ${ }^{(37)}$, our group of patients are comparable with the average OSA patients in Sweden in terms of age, gender, BMI, AHI, ODI, ESS and cardiometabolic diseases. Nonetheless, there are limitations to consider. The relatively short time to follow-up may have affected the results, since some patients may need a longer time to adjust to CPAP treatment. When evaluating SNOT-22 scores and PNIF values, it would have been desirable to have a control group. Furthermore, additional objective nasal measurements, such as rhinomanometry or acoustic rhinometry, and a clinical examination of the nose and sinuses or CT scan would also have added further information.

\section{Conclusion}

We conclude that SNOT-22 scores are elevated in OSA patients, indicating a considerable sinonasal disease burden. SNOT-22 scores improve with good CPAP adherence. We also conclude that a low PNIF value, $<100 \mathrm{l} / \mathrm{min}$, is able to predict poor initial CPAP adherence. A low PNIF value should not exclude patients from initiating CPAP treatment but could be used as an incentive to treat nasal obstruction to optimise the treatment.
We propose that both the SNOT-22 and PNIF could be valuable tools in the evaluation of OSA patients and in the management of CPAP treatment.

\section{Acknowledgements}

The authors wish to convey their gratitude to Pernilla Sahlstrand-Johnson, MD, PhD, Department of Oto-Rhino-Laryngology, Head-and-Neck Surgery, Lund University, Skåne University Hospital, Malmö, Sweden, for permission to use the Swedish version of the SNOT-22 and for her useful advice, and to Maria Hillerdal, MD, PhD, Department of Surgical Sciences, Otorhinolaryngology, Head and Neck Surgery, Uppsala University, Uppsala, Sweden for her generous contribution of PNIF meters.

The study was funded by the Swedish Heart and Lung Foundation, the Uppsala County Association against Heart and Lung Diseases, the Bror Hjerpstedt Foundation, the ACTA Otolaryngologica Foundation, Uppsala University, ALF fundings and FoU fundings.

\section{Authorship contribution}

$\mathrm{CB}$ contributed with the design of the study and the acquisition, analysis and interpretation of data and manuscript writing. LJ, $\mathrm{MH}$ and $\mathrm{CJ}$ contributed with the design of the study, interpretation of data and critical revision of the manuscript. JTH contributed with the analysis and interpretation of data, and critical revision of the manuscript. EL contributed with the design of the study, analysis and interpretation of data and critical revision of the manuscript.

\section{Conflict of interest}

The authors have no conflicts of interest to declare.

\section{References}

1. Oliveira MC, Tufik S, Haddad FL, SantosSilva R, Gregorio LC, Bittencourt L. Systematic Evaluation of the Upper Airway in a Sample Population: Factors Associated with Obstructive Sleep Apnea Syndrome. Otolaryngol-Head Neck Surg 2015;153(4):663-70.

2. Brander PE, Soirinsuo $M$, Lohela $P$. Nasopharyngeal symptoms in patients with obstructive sleep apnea syndrome. Effect of nasal CPAP treatment. Respiration. 1999;66(2):128-35

3. Lojander J, Brander PE, Ammala K Nasopharyngeal symptoms and nasal continuous positive airway pressure therapy in obstructive sleep apnoea syndrome. Acta Otolaryngologica. 1999;119(4):497-502.

4. Varendh M, Andersson M, Bjornsdottir $E_{\text {, }}$ Hrubos-Strom H, Johannisson A, Arnardottir ES, et al. Nocturnal nasal obstruction is frequent and reduces sleep quality in patients with obstructive sleep apnea. J Sleep Res.

\section{8;27(4):e12631.}

5. Moxness $M H$, Bugten $V$, Thorstensen WM Nordgard S, Bruskeland G. A comparison of minimal cross sectional areas, nasal volumes and peak nasal inspiratory flow between patients with obstructive sleep apnea and healthy controls. Rhinology. 2016:54(4):342-7.

6. Morley AD, Sharp HR. A review of sinonasal outcome scoring systems - which is best? Clin Otolaryngol. 2006;31(2):103-9.

7. Kuan EC, Tajudeen BA, Peng KA, Wang MB. Sinonasal outcomes in obstructive sleep apnea syndrome. Laryngoscope. 2015;125(11):2617-20.

8. Weaver TE, Grunstein RR. Adherence to continuous positive airway pressure therapy: the challenge to effective treatment. Proc Am Thorac Soc. 2008;5(2):173-8.

9. Franklin KA, Lindberg E. Obstructive sleep apnea is a common disorder in the population-a review on the epidemiology of sleep apnea. J Thorac Dis. 2015:7(8):1311-22.
10. Ottaviano G, Fokkens WJ. Measurements of nasal airflow and patency: a critical review with emphasis on the use of peak nasal inspiratory flow in daily practice. Allergy. 2016;71(2):162-74

11. Cho SI, Hauser R, Christiani DC Reproducibility of nasal peak inspiratory flow among healthy adults: assessment of epidemiologic utility. Chest. 1997;112(6):1547-53.

12. Starling-Schwanz $R$, Peake $H L$, Salome CM, Toelle BG, Ng KW, Marks GB, et al. Repeatability of peak nasal inspiratory flow measurements and utility for assessing the severity of rhinitis. Allergy. 2005;60(6):795800.

13. Teixeira RU, Zappelini CE, Alves FS, da Costa EA. Peak nasal inspiratory flow evaluation as an objective method of measuring nasal airflow. Braz J Otorhinolaryngol. 2011;77(4):473-80.

14. Weaver TE, Maislin G, Dinges DF, Bloxham T, George CF, Greenberg H, et al. Relationship 
between hours of CPAP use and achieving normal levels of sleepiness and daily functioning. Sleep. 2007;30(6):711-9.

15. Ottaviano G, Scadding GK, lacono V, Scarpa B, Martini A, Lund VJ. Peak nasal inspiratory flow and peak expiratory flow. Upright and sitting values in an adult population. Rhinology. 2016;54(2):160-3.

16. Ottaviano G, Scadding GK, Coles S, Lund VJ Peak nasal inspiratory flow; normal range in adult population. Rhinology. 2006;44(1):32 5.

17. Hopkins C, Gillett S, Slack R, Lund VJ, Browne JP. Psychometric validity of the 22-item Sinonasal Outcome Test. Clin Otolaryngol. 2009;34(5):447-54.

18. Piccirillo JF, Merritt MG, Jr., Richards ML. Psychometric and clinimetric validity of the 20-Item Sino-Nasal Outcome Test (SNOT-20). Otolaryngol Head Neck Surg. 2002;126(1):41-7.

19. Browne JP, Hopkins C, Slack R, Cano SJ. The Sino-Nasal Outcome Test (SNOT): can we make it more clinically meaningful? Otolaryngol Head Neck Surg. 2007;136(5):736-41

20. Zigmond AS, Snaith RP. The hospital anxiety and depression scale. Acta Psychiatr Scand. 1983;67(6):361-70.

21. Johns MW. A new method for measuring daytime sleepiness: the Epworth sleepiness scale. Sleep. 1991;14(6):540-5

22. Sahlstrand-Johnson P, Ohlsson B, Von Buchwald C, Jannert M, Ahlner-Elmqvist M. A multi-centre study on quality of life and absenteeism in patients with CRS referred for endoscopic surgery. Rhinology. 2011;49(4):420-8

23. Fokkens WJ, Lund VJ, Mullol J, Bachert C Alobid I, Baroody F, et al. European Position Paper on Rhinosinusitis and Nasal Polyps 2012. Rhinology Supplement. 2012(23):3 p preceding table of contents, 1-298.
24. Farhood Z, Schlosser RJ, Pearse ME, Storck KA, Nguyen SA, Soler ZM. Twenty-two-item Sino-Nasal Outcome Test in a control population: a cross-sectional study and systematic review. Int Forum Allergy Rhinol. 2016;6(3):271-7.

25. Lundback B, Backman H, Lotvall J, Ronmark E. Is asthma prevalence still increasing? Expert Rev Respir Med. 2016;10(1):39-51.

26. Bengtsson $C$, Lindberg $E$, Jonsson L, Holmstrom M, Sundbom F, Hedner J, et al. Chronic rhinosinusitis impairs sleep quality - results of the GA2LEN study. Sleep. 2016

27. Sahlstrand-Johnson P, Hopkins C, Ohlsson $B$, Ahlner-Elmqvist M. The effect of endoscopic sinus surgery on quality of life and absenteeism in patients with chronic rhinosinuitis - a multi-centre study. Rhinology. 2017;55(3):251-61.

28. Hoven KM, Aarstad HJ, Bjorvatn B, Lundemo EH, Steinsvag SK. Correlation between Excessive Daytime Sleepiness (EDS) and self-reported and objective nasal characteristics. Rhinology. 2018;56(4):316-22.

29. Sugiura T, Noda A, Nakata S, Yasuda Y, Soga $T$, Miyata $S$, et al. Influence of nasal resistance on initial acceptance of continuous positive airway pressure in treatment for obstructive sleep apnea syndrome. Respiration. 2007;74(1):56-60.

30. Nakata S, Noda A, Yagi H, Yanagi E, Mimura T, Okada T, et al. Nasal resistance for determinant factor of nasal surgery in CPAP failure patients with obstructive sleep apnea syndrome. Rhinology. 2005;43(4):296-9.

31. Li HY, Engleman $H, H s u C Y$, Izci B, Vennelle M, Cross M, et al. Acoustic reflection for nasal airway measurement in patients with obstructive sleep apnea-hypopnea syndrome. Sleep. 2005;28(12):1554-9.

32. Blomgren K, Simola $M$, Hytonen $M$, Pitkaranta A. Peak nasal inspiratory and expiratory flow measurements--prac- tical tools in primary care? Rhinology. 2003:41(4):206-10

33. Bouzgarou MD, Ben Saad H, Chouchane A, Cheikh IB, Zbidi A, Dessanges JF, et al. North African reference equation for peak nasal inspiratory flow. J Laryngol Otol. 2011;125(6):595-602.

34. Klossek JM, Lebreton JP, Delagranda A, Dufour X. PNIF measurement in a healthy French population. A prospective study about 234 patients. Rhinology. 2009;47(4):389-92.

35. Nsair A, Hupin D, Chomette S, Barthelemy JC, Roche F. Factors Influencing Adherence to Auto-CPAP: An Observational Monocentric Study Comparing Patients With and Without Cardiovascular Diseases. Frontiers Neurol. 2019;10:801.

36. https://stratum.registercentrum.se.

37. https://www.ucr.uu.se/swedevox/.
Caroline Bengtsson

Department of Surgical Sciences

Otorhinolaryngology

Head and Neck Surgery

Uppsala University

Akademiska Sjukhuset, ing 70, 1 tr

SE-751 85 Uppsala

Sweden

Tel: +46 (0) 70-579 1676

E-mail:

caroline.bengtsson@surgsci.uu.se 